\title{
Caryophyllene sesquiterpenoids from a fungicolous isolate of Pestalotiopsis disseminata (Thuem.)
}

Stephen T. Deyrup ${ }^{\dagger}$, Dale C. Swenson ${ }^{\dagger}$, James B. Gloer ${ }^{\dagger} *$, and Donald T. Wicklow ${ }^{\ddagger}$

Department of Chemistry, University of Iowa, Iowa City, Iowa 52242 and Mycotoxin Research Unit, Agricultural Research Service, National Center for Agricultural Utilization Research, USDA, Peoria, Illinois 61604

\section{List of Supporting Information}

Figure S1. ${ }^{1} \mathrm{H}$ NMR spectrum of 6-hydroxypunctaporonin E $\left(\mathbf{1} ; 300 \mathrm{MHz}, \mathrm{CDCl}_{3}\right)$

Figure S2. ${ }^{13} \mathrm{C}$ NMR spectrum of 6-hydroxypunctaporonin $\mathrm{E}\left(\mathbf{1} ; 75 \mathrm{MHz}, \mathrm{CDCl}_{3}\right)$

Figure S3. ${ }^{1} \mathrm{H}$ NMR spectrum of 6-hydroxypunctaporonin $\mathrm{B}\left(\mathbf{2} ; 300 \mathrm{MHz}, \mathrm{CDCl}_{3}\right)$

Figure S4. ${ }^{13} \mathrm{C}$ NMR spectrum of 6-hydroxypunctaporonin $\mathrm{B}\left(\mathbf{1} ; 75 \mathrm{MHz}, \mathrm{CDCl}_{3}\right)$

Figure S5. ${ }^{1} \mathrm{H}$ NMR spectrum of 6-hydroxypunctaporonin A $\left(\mathbf{3} ; 300 \mathrm{MHz}, \mathrm{CD}_{3} \mathrm{OD}\right)$

Figure S6. ${ }^{13} \mathrm{C}$ NMR spectrum of 6-hydroxypunctaporonin $\mathrm{A}\left(\mathbf{3} ; 75 \mathrm{MHz}, \mathrm{CD}_{3} \mathrm{OD}\right)$

Figure S7. ${ }^{1} \mathrm{H}$ NMR spectrum of 12-(4-bromobenzoyl)-6-hydroxypunctaporonin E (4; $\left.300 \mathrm{MHz}, \mathrm{CDCl}_{3}\right)$ 


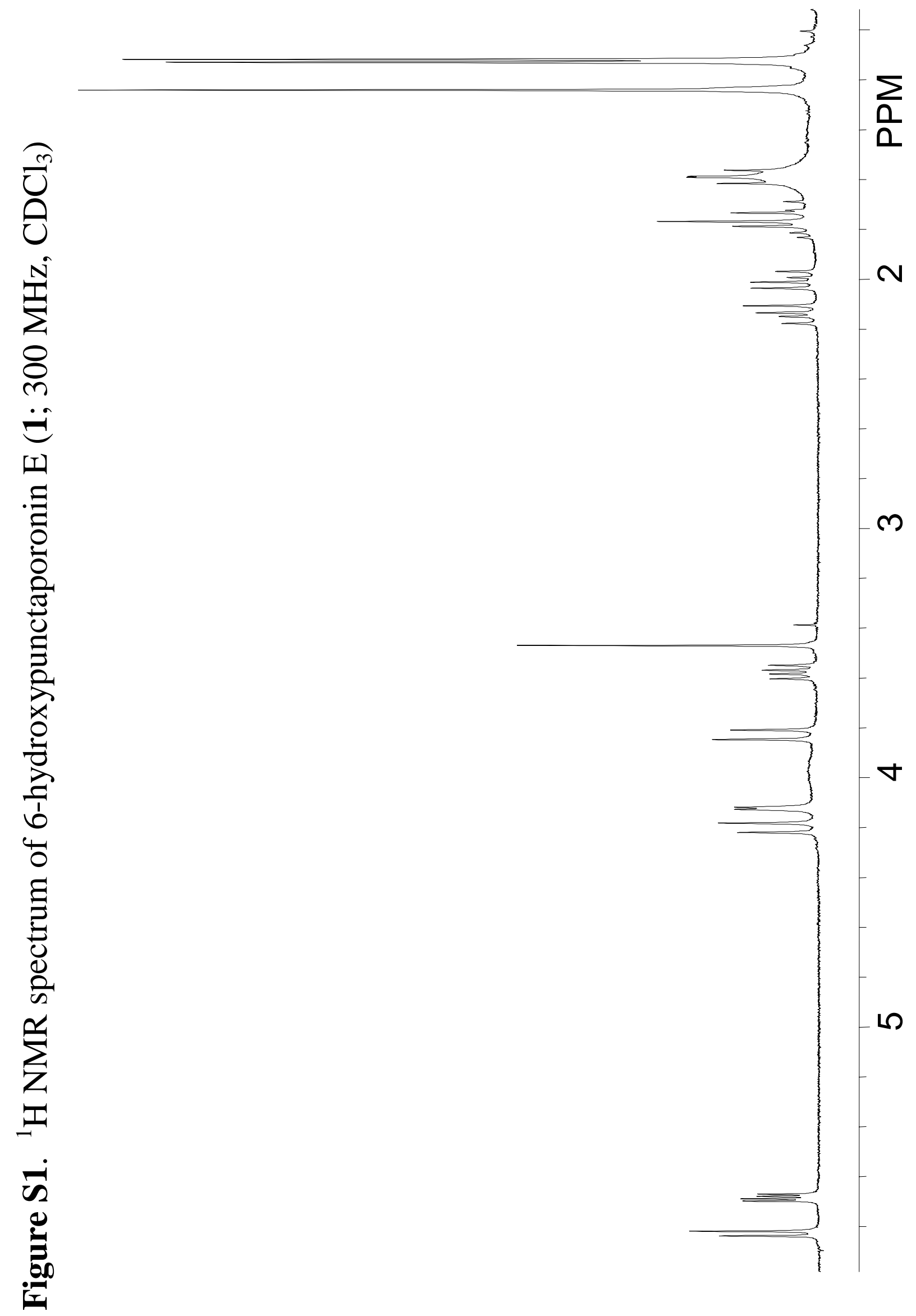




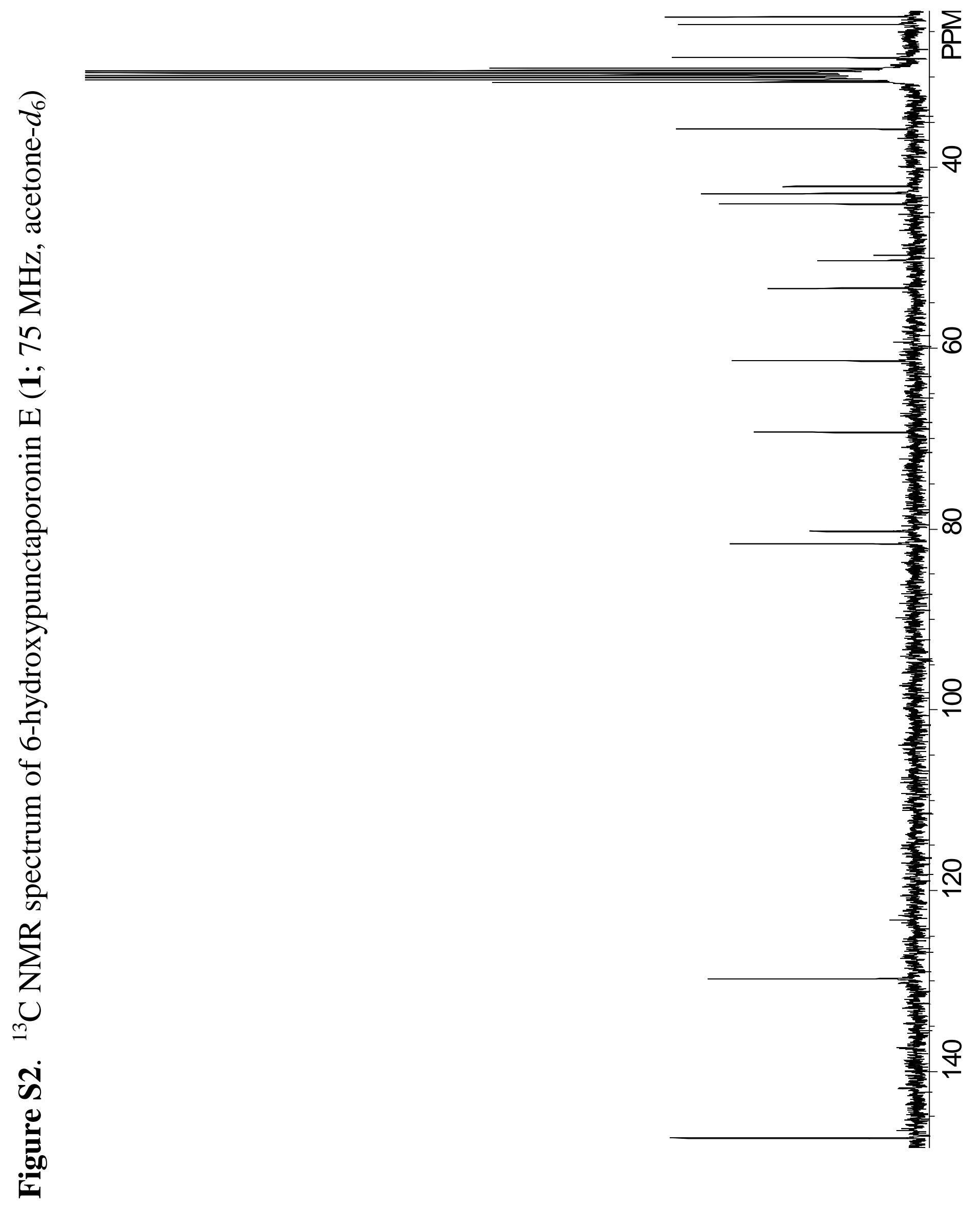




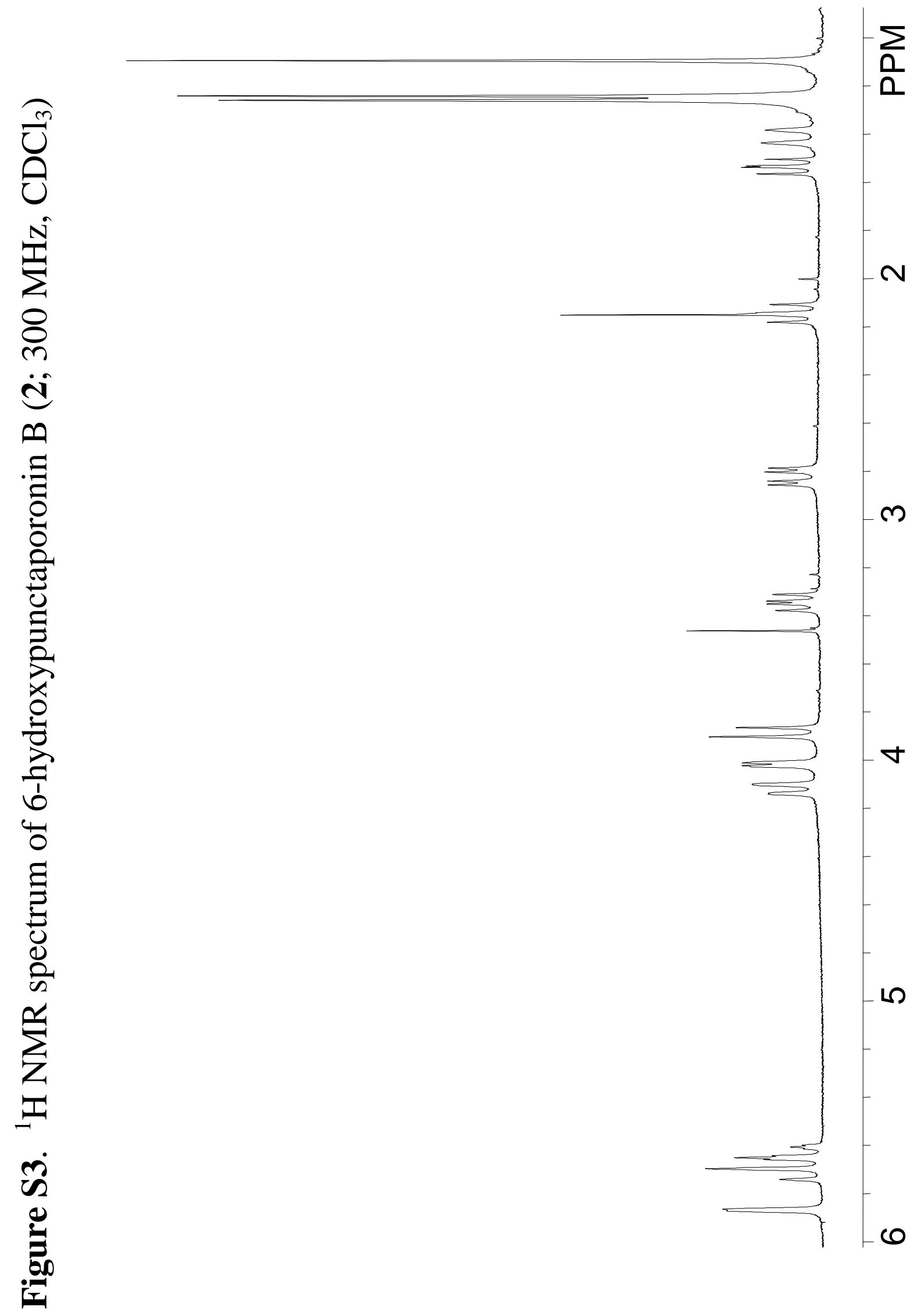



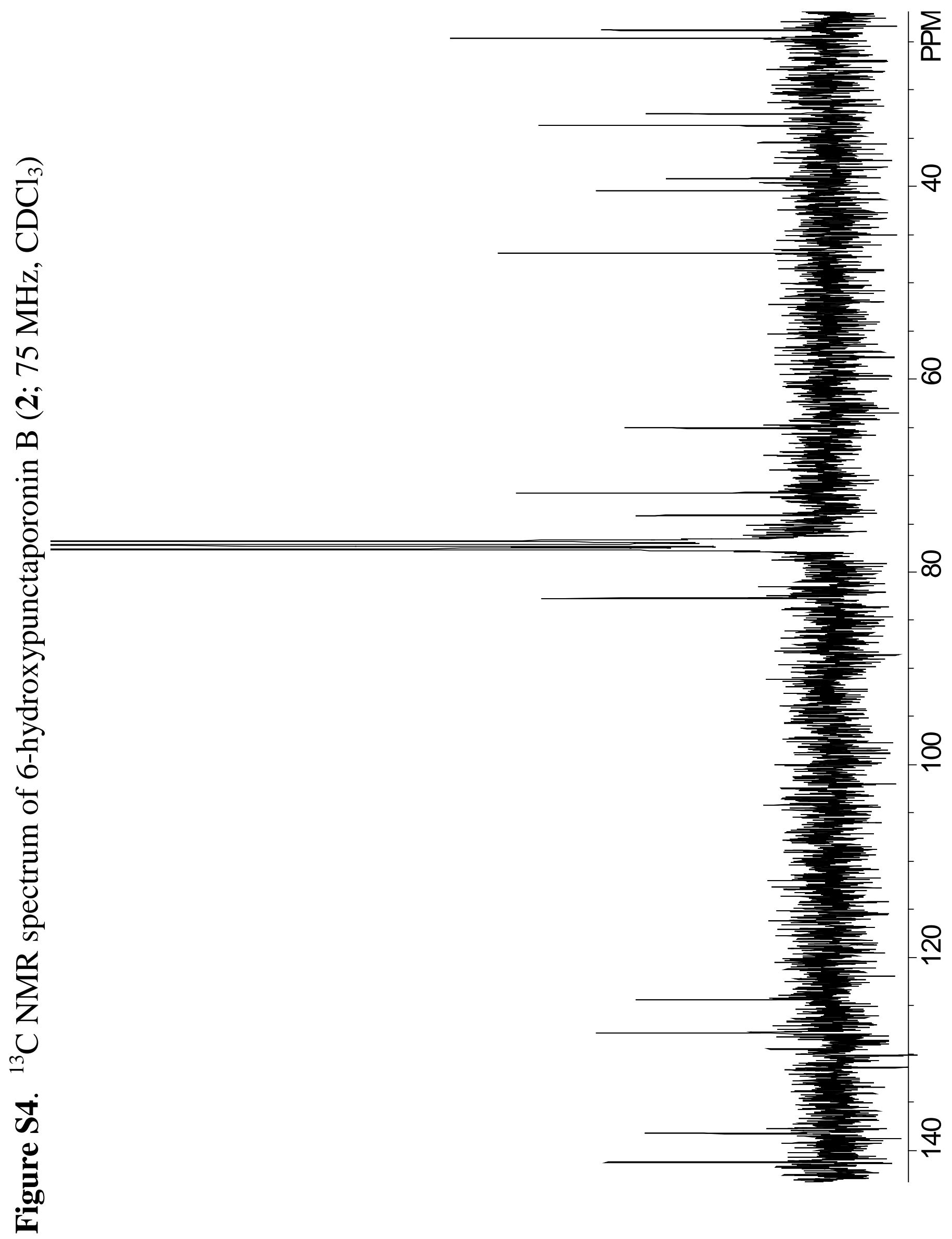


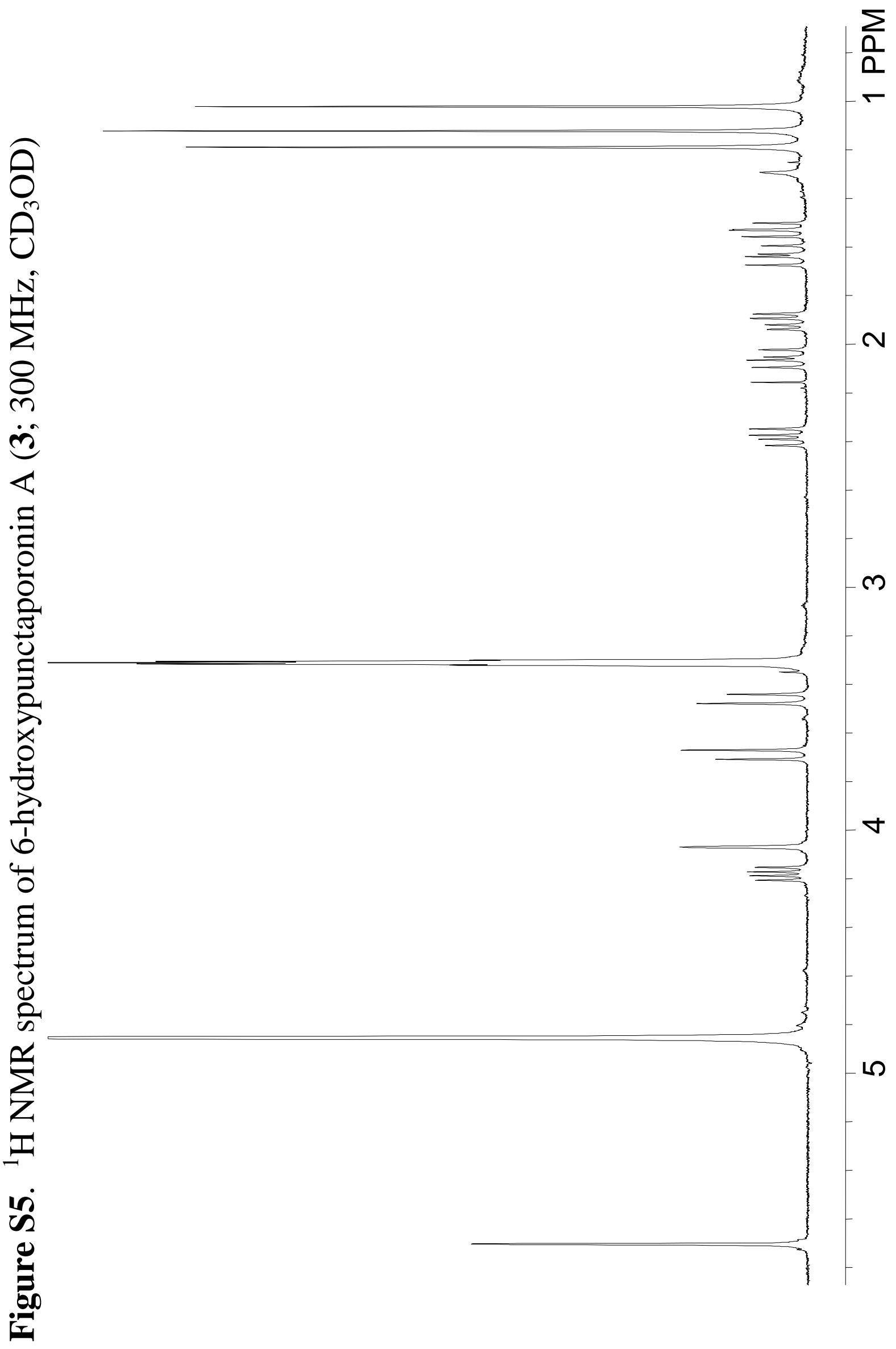




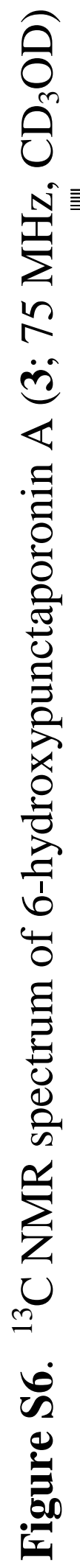




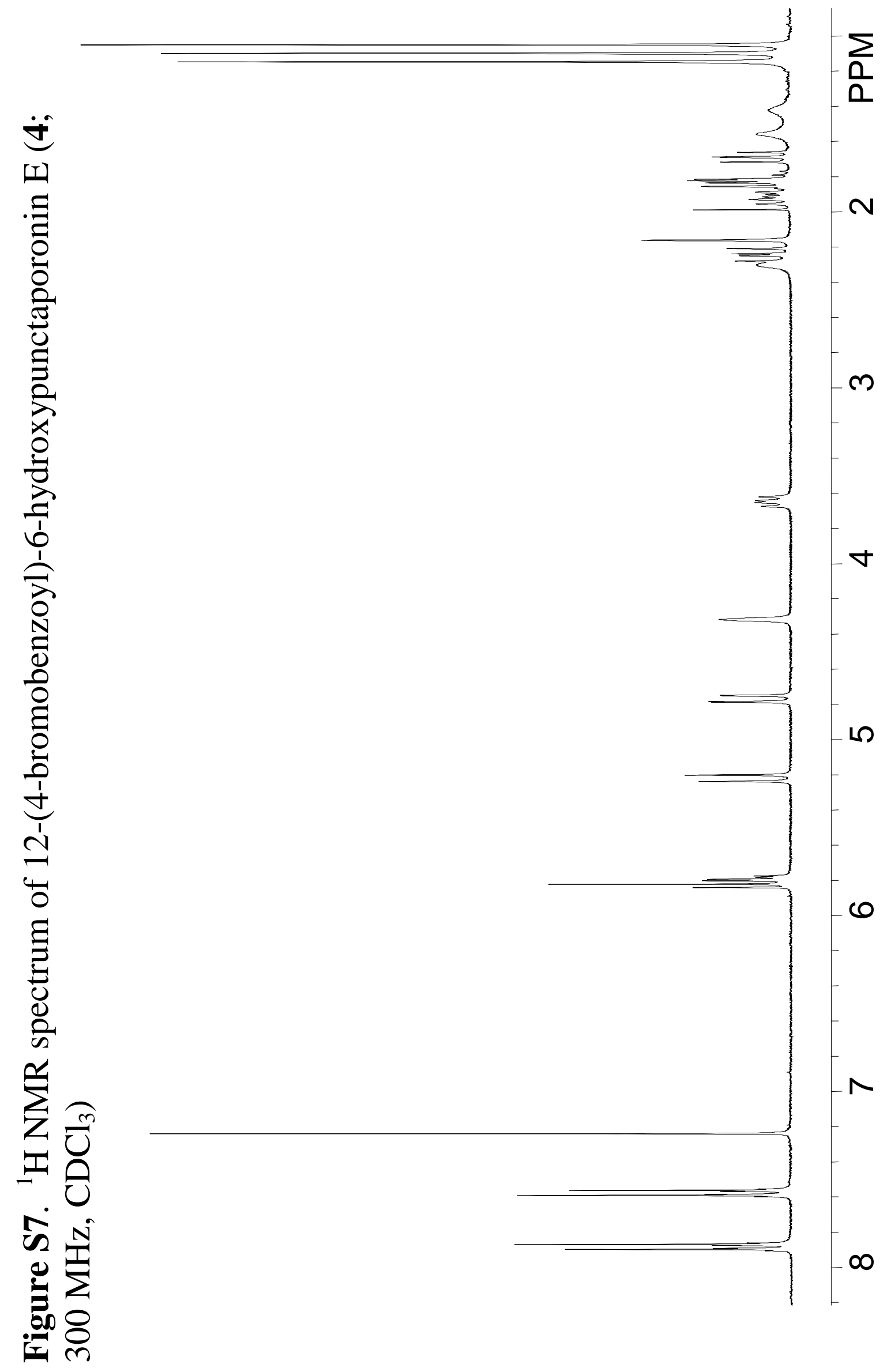

